\title{
RIEMANNIAN MANIFOLDS ISOSPECTRAL ON FUNCTIONS BUT NOT ON 1-FORMS
}

\author{
CAROLYN S. GORDON
}

\section{Introduction}

For $(M, g)$ a compact Riemannian manifold, let $\operatorname{spec}^{p}(M, g)$ denote the collection of eigenvalues, with multiplicities, of the associated Laplace-Beltrami operator acting on the space of smooth $p$-forms on $M, p=0,1,2, \cdots, \operatorname{dim}(M)$. Two manifolds $(M, g)$ and $\left(M^{\prime}, g^{\prime}\right)$ will be said to be $p$-isospectral if $\operatorname{spec}^{p}(M, g)=\operatorname{spec}^{p}\left(M^{\prime}, g^{\prime}\right)$. Note that 0 -isospectral manifolds are generally called "isospectral" in the literature. It is well known that $\operatorname{spec}^{0}(M, g)$ (i.e. the spectrum on functions) contains considerable information about the geometry of $(M, g)$. Other information is known to be contained in the $p$-spectra for

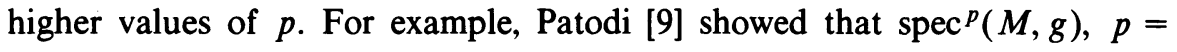
$0,1,2$, together determine whether $(M, g)$ has constant scalar curvature, whether it is Einstein, and whether it has constant sectional curvature. It would be of interest to determine whether for each $k$, the collection of all $\operatorname{spec}^{p}(M, g), p=0, \cdots, k$, contains more information than $\operatorname{does}^{\operatorname{spec}^{p}}(M, g)$, $p=0, \cdots, k-1$. The purpose of this article is to give an affirmative answer when $k=1$, i.e., we give examples of manifolds which are 0 -isospectral but not 1 -isospectral.

The manifolds in our examples are Riemannian Heisenberg manifolds, i.e., compact quotients $\Gamma \backslash H^{n}$ of the $(2 n+1)$-dimensional Heisenberg group, with metrics $g$ induced by left-invariant metrics on $H_{n}$. In [5], we gave sufficient conditions for two Riemannian Heisenberg manifolds to be 0 -isospectral and constructed many examples. We will see that some of these examples are $p$-isospectral for all $p$ while others are not 1 -isospectral. We give evidence suggesting that these are the only two possibilities, i.e. that once the manifolds in these examples are 1-isospectral, they are also $p$-isospectral for all $p$. We

Received February 10, 1986. The research for this work was partially supported by National Science Foundation Grant DMS-8502084. 
also attempt to distinguish geometrically those examples which are only 0 -isospectral from those which are also 1-isospectral.

The paper is organized as follows: In $\S 1$, we discuss one method (needed later) for proving that two manifolds are $p$-isospectral for all $p$. We also make a few side remarks concerning Vignéras' examples [11] of 0 -isospectral manifolds which are not isometric. $\$ 2$ reviews the necessary facts about Riemannian Heisenberg manifolds, establishes the notation used throughout the paper, and gives a few preliminary results. In $\$ \S 3-5$, we compare the spectra of Riemannian Heisenberg manifolds $\left(\Gamma \backslash H_{n}, g\right)$ and $\left(\Gamma^{\prime} \backslash H_{n}, g\right)$ under the hypothesis that $\Gamma$ and $\Gamma^{\prime}$ have the same intersection with the center of $H_{n}$. In Theorem 3.2 , we give a necessary and sufficient condition (P0) for such a pair to be 0 -isospectral. (The sufficiency of a slightly stronger condition was proven in [5].) In $\$ \S 4$ and 5 we assume (P0) to be satisfied and give additional sufficient conditions (P1) for the manifolds to be 1-isospectral (Theorem 4.3) and (P2) for them to be $p$-isospectral for all $p$ (Theorem 5.2). In Theorem 4.4, we see that under an additional hypothesis - and we conjecture always-(P1) is also necessary for the manifolds to be 1-isospectral. Proposition 5.4 compares conditions (P1) and (P2) and, together with Theorem 4.4, motivates our conjecture that 0 - and 1-isospectral Heisenberg manifolds must be $p$ isospectral for all $p$. Finally in $\S 6$, we restrict our attention to groups $\Gamma$ and $\Gamma^{\prime}$ for which the conditions (P0), (P1), and (P2) have a particularly simple form, enabling us to construct specific examples of 0 -isospectral Heisenberg manifolds. We consider four such examples and compare their spectra on forms and also compare their fundamental groups. These examples illustrate that for 0 -isospectral Heisenberg manifolds, the questions of whether the manifolds are 1-isospectral and whether their fundamental groups are isomorphic are independent.

\section{A method for proving manifolds are isospectral}

Let $G$ be a simply-connected Lie group, $g$ a left-invariant Riemannian metric on $G$, and $\Gamma$ a uniform discrete subgroup of $G$. ("Uniform" means that $\Gamma \backslash G$ is compact.) The metric $g$ induces a Riemannian metric, again denoted $g$, on $\Gamma \backslash G$ so that $(G, g)$ is a Riemannian covering of $(\Gamma \backslash G, g)$. The space $L^{2}(\Gamma \backslash G)$ is identified with

$$
L^{2}(\Gamma \backslash G)=\left\{f \in L_{\text {loc }}^{2}(G): f(\gamma x)=f(x) \text { for all } \gamma \in \Gamma, x \in G\right\} .
$$

The Lie algebra $g$ of $G$ may be identified both with the tangent space $T_{e}(G)$ (so $g$ defines an inner product on $g$ ) and with the space of left-invariant vector fields on $G$. Such vector fields may also be viewed as vector fields on $\Gamma \backslash G$. 
We view elements of $\Lambda^{p}\left(g^{*}\right)$ both as left-invariant $p$-forms on $G$ and also as forms on $\Gamma \backslash G$. The space of square-integrable $p$-forms is given by

$$
L^{2}(\Gamma \backslash G) \otimes \Lambda^{p}\left(g^{*}\right) .
$$

The Laplacian of $(\Gamma \backslash G, g)$ acting on smooth functions is given by $\Delta=$ $-\sum_{i=1}^{n} X_{i}^{2}$, where $\left\{X_{1}, \cdots, X_{n}\right\}$ is an orthonormal basis of $g$ relative to the inner product defined by $g$. Let $\rho$ denote the right action of $G$ on $L^{2}(\Gamma \backslash G)$, i.e.

$$
\rho(x) f(y)=f(y x) .
$$

Since $X_{i} f=d f\left(x \exp t X_{i}\right) / d t_{\mid t=0}$, the extension of $\Delta$ to $L^{2}(\Gamma \backslash G)$ is given by

$$
\Delta=-\sum_{i=1}^{n}\left(\rho_{*}\left(X_{i}\right)\right)^{2} .
$$

Note that if $\Phi \in \operatorname{Aut}(G)$ and $\Phi^{*} g=g$, then $\left\{\Phi_{*} X_{1}, \cdots, \Phi_{*} X_{n}\right\}$ is another orthonormal basis of $g$ and hence we also have

$$
\Delta=-\sum_{i=1}^{n}\left((\rho \circ \Phi)_{*} X_{i}\right)^{2}
$$

Now let $\Gamma^{\prime}$ be a second uniform discrete subgroup of $G$. Denote by $\Delta^{\prime}$ and $\rho^{\prime}$ the Laplacian of $\left(\Gamma^{\prime} \backslash G, g\right)$ and the right action of $G$ on $L^{2}\left(\Gamma^{\prime} \backslash G\right)$, respectively.

(1.1) Definition. Let $(\Gamma \backslash G, g)$ and $\left(\Gamma^{\prime} \backslash G, g\right)$ be as above, let $\mathscr{H}$ be a $\rho$-invariant subspace of $L^{2}(\Gamma \backslash G)$, and $\mathscr{H}^{\prime}$ a $\rho^{\prime}$-invariant subspace of $L^{2}\left(\Gamma^{\prime} \backslash G\right)$. We say $\mathscr{H}$ is $g$-equivalent to $\mathscr{H}^{\prime}$ if there exists $\Phi \in \operatorname{Aut}(G)$ such that $g=\Phi^{*} g$ and such that $\rho$ acting on $\mathscr{H}$ is equivalent to $\rho^{\prime} \circ \Phi$ acting on $\mathscr{H}^{\prime}$.

(1.2) Theorem. Let $(\Gamma \backslash G, g)$ and $\left(\Gamma^{\prime} \backslash G, g\right)$ be as above. Suppose $\mathscr{H}$ is a $\rho$-invariant subspace of $L^{2}(\Gamma \backslash G), \mathscr{H}^{\prime}$ is a $\rho^{\prime}$-invariant subspace of $L^{2}\left(\Gamma^{\prime} \backslash G\right)$, and $\mathscr{H}$ is g-equivalent to $\mathscr{H}^{\prime}$. Then the spaces $\mathscr{H} \otimes \Lambda^{p}\left(\mathrm{~g}^{*}\right)$ and $\mathscr{H}^{\prime} \otimes \Lambda^{p}\left(\mathrm{~g}^{*}\right)$ of $p$-forms are invariant under the action of the Laplacians $\Delta$ and $\Delta^{\prime}$, respectively, and the Laplacians restricted to these subspaces are isospectral.

(1.3) Corollary. Suppose there exist decompositions $L^{2}(\Gamma \backslash G)=\bigoplus_{\alpha \in A} \mathscr{H}_{\alpha}$ and $L^{2}\left(\Gamma^{\prime} \backslash G\right)=\bigoplus_{\alpha \in A} \mathscr{H}_{\alpha}^{\prime}$ into $\rho$-invariant (respectively $\rho^{\prime}$-invariant) subspaces such that for each $\alpha, \mathscr{H}_{\alpha}$ is g-equivalent to $\mathscr{H}_{\alpha}^{\prime}$. Then $(\Gamma \backslash G, g)$ is p-isospectral to $\left(\Gamma^{\prime} \backslash G, g\right)$ for all $p$.

Proof. A special case of the theorem and corollary (with the automorphisms "almost inner") is proved in [4]; the additional hypotheses are not needed in the proof. 
The method of Corollary 1.3 was used in [1] and [4]. Most methods which have been used to construct 0 -isospectral manifolds similarly involve representation theoretic or algebraic techniques which force the manifolds to be $p$-isospectral for all $p$ as well as 0-isospectral. (See Sunada's examples [10] and also Gilkey's proof [2] that Ikeda's examples [8] of 0-isospectral manifolds are actually $p$-isospectral for all $p$.)

We will see that in the case of various isospectral Heisenberg manifolds $\left(\Gamma \backslash H_{n}, g\right)$ and $\left(\Gamma^{\prime} \backslash H_{n}, g\right)$, the spaces $L^{2}\left(\Gamma \backslash H_{n}\right)$ and $L^{2}\left(\Gamma^{\prime} \backslash H_{n}\right)$ admit decompositions of the form

$$
L^{2}\left(\Gamma \backslash H_{n}\right)=\mathscr{H}_{\Gamma, 1} \oplus \mathscr{H}_{\Gamma, 2}
$$

such that $H_{\Gamma, 2}$ is $g$-equivalent to $\mathscr{H}_{\Gamma, 2}^{\prime}$. However, $\mathscr{H}_{\Gamma, 1}$ need not be $q$ equivalent to $\mathscr{H}_{\Gamma^{\prime}, 1}$ even though the Laplacians are isospectral on these spaces. When these spaces are not $g$-equivalent, the manifolds will in general not be 1-isospectral.

(1.4) Remark. Vignéras [11] used similar methods to construct isospectral compact hyperbolic manifolds. Let $G$ be a direct product $\operatorname{SL}(2, \mathbf{R})^{r} \times \operatorname{SL}(2, \mathbf{C})^{s}$. She constructs pairs of uniform discrete subgroups $\Gamma$ and $\Gamma^{\prime}$ such that the right actions of $G$ on $L^{2}(\Gamma \backslash G)$ and $L^{2}\left(\Gamma^{\prime} \backslash G\right)$ are equivalent. As she points out, the manifolds $\Gamma \backslash G / K$ and $\Gamma^{\prime} \backslash G / K$ are then 0 -isospectral, where $K$ is a maximal compact subgroup of $G$ and $G / K$ is given the symmetric space metric. By Corollary 1.3, we see that for any left-invariant Riemannian metric $g$ on $G$, the manifolds $(\Gamma \backslash G, g)$ and $\left(\Gamma^{\prime} \backslash G, g\right)$ are $p$-isospectral for all $p$.

\section{Riemannian Heisenberg manifolds}

By a Riemannian Heisenberg manifold, we shall mean a manifold of the form ( $\left.\Gamma \backslash H_{n}, g\right)$, where $H_{n}$ is the $(2 n+1)$-dimensional Heisenberg group (see (2.1) below), $\Gamma$ is a uniform discrete subgroup, and $g$ is a Riemannian metric whose lift to $H_{n}$, again denoted $g$, is left-invariant. The isometry classes of Riemannian Heisenberg manifolds were classified in [5] by specifying a collection $\mathscr{S}$ of Riemannian Heisenberg manifolds, including at least one from each isometry class, and specifying a necessary and sufficient condition for two members of $\mathscr{S}$ to be isometric. The particular collection $\mathscr{S}$ of representative manifolds was convenient for the computations carried out in [5]; however the main results of the present paper can be stated more simply if we use a different collection $\mathscr{T}$. We will define $\mathscr{T}$ below and indicate how to find for each manifold in $\mathscr{T}$ (or $\mathscr{S}$ ) an isometric manifold in $\mathscr{S}$ (or in $\mathscr{T}$, respectively). This will allow us to translate the results of [5] to the new setting whenever necessary. We also describe the Ricci tensors of the elements of $\mathscr{T}$. 
(2.1) The Heisenberg group and Lie algebra. The $(2 n+1)$-dimensional Heisenberg group is the subgroup $H_{n}=\left\{h(x, y, t): x, y \in \mathbf{R}^{n}, t \in \mathbf{R}\right\}$ of $\mathrm{GL}(n+2, \mathbf{R})$, where

$$
h(x, y, t)=\left(\begin{array}{ccccc}
1 & x_{1} & \cdots & x_{n} & t \\
& 1 & 0 & & y_{1} \\
& & \ddots & & \vdots \\
& 0 & & 1 & y_{n} \\
& & & & 1
\end{array}\right)
$$

$H_{n}$ is diffeomorphic to $\mathbf{R}^{2 n} \times \mathbf{R}$. The factor $\mathbf{R}=\{h(0,0, t): t \in \mathbf{R}\}$ is the center of $H_{n}$. Multiplication is given by

$$
h(x, y, t) h\left(x^{\prime}, y^{\prime}, t^{\prime}\right)=h\left(x+x^{\prime}, y+y^{\prime}, t+t^{\prime}+x \cdot y^{\prime}\right),
$$

where $x \cdot y^{\prime}$ is the standard dot product in $\mathbf{R}^{n}$.

The Lie algebra $\mathfrak{h}_{n}$ of $H_{n}$ has basis

$$
\mathscr{B}=\left\{X_{1}, \cdots, X_{n}, Y_{1}, \cdots, Y_{n}, Z\right\}
$$

satisfying $\left[X_{i}, Y_{i}\right]=Z$ with all other brackets of basis elements equal to zero. Thus $\mathbf{R} Z$ is the center of $\mathfrak{h}_{n}$. If we write

$$
X(x, y, t)=\sum_{i=1}^{n}\left(x_{i} X_{i}+y_{i} Y_{i}\right)+t Z
$$

then $\mathfrak{h}_{n}$ may be viewed as a matrix algebra with

$$
X(x, y, t)=\left(\begin{array}{ccccc}
0 & x_{1} & \cdots & x_{n} & t \\
& & & & y_{1} \\
& & & & \vdots \\
& 0 & & & y_{n} \\
& & & & 0
\end{array}\right) .
$$

As a vector space $\mathfrak{h}_{\mathbf{n}} \cong \mathbf{R}^{2 n}+\mathbf{R} Z$. We thus view $\mathbf{R}^{2 n}$ as a subspace of $\mathfrak{h}_{h}$.

The group exponential satisfies

$$
\exp (X(x, y, t))=h\left(x, y, t+\frac{1}{2} x \cdot y\right)
$$

with inverse $\log h(x, y, t)=X\left(x, y, t-\frac{1}{2} x \cdot y\right)$.

(2.4) Automorphisms of $H_{n}$. We will identify each automorphism $\Phi$ of $H_{n}$ with the matrix of its differential $\Phi_{*}$ relative to the standard basis $\mathscr{B}$ of $\mathfrak{h}_{n}$. Let

$$
J=\left[\begin{array}{cc}
0 & I_{n} \\
-I_{n} & 0
\end{array}\right],
$$

$$
\widetilde{\mathrm{Sp}}(n, \mathbf{R})=\left\{\beta \in \mathrm{GL}(2 n, \mathbf{R}):{ }^{t} \beta J \beta=\varepsilon J \quad \text { with } \varepsilon= \pm 1\right\}
$$


As discussed in [5], Aut $\left(H_{n}\right)$ is the set of all matrices $\Phi$ of the form

$$
\Phi=\left[\begin{array}{cc}
a \beta & 0 \\
w & a^{2} \varepsilon
\end{array}\right],
$$

where $a \in \mathbf{R}^{*}, w \in \mathbf{R}^{2 n}$, and $\beta \in \widetilde{\mathrm{Sp}}(n, \mathbf{R})$.

(2.5) Left-invariant matrices. Each left-invariant Riemannian metric $g$ on $H_{n}$ is uniquely determined by the associated inner product on the tangent space at the identity element $e$. Since $T_{e}\left(H_{n}\right) \cong \mathfrak{h}_{n}$, we will identify $g$ with the matrix of this inner product relative to the standard basis $\mathscr{B}$ of $\mathfrak{h}_{n}$. We will say $g$ is an $\mathscr{S}$-metric if

$$
g=\left(\begin{array}{cc} 
& 0 \\
g_{0} & \vdots \\
& 0 \\
0 \cdots & d^{2}
\end{array}\right)
$$

for some positive definite $2 n \times 2 n$ matrix $g_{0}$ and $d \in \mathbf{R}^{+}$, and $g$ is a $\mathscr{T}$-metric if

$$
g=\left(\begin{array}{ccccccc}
a_{1} & & & & & & \\
& \ddots & & & 0 & & \\
& & a_{n} & & & & \\
& & & a_{1} & & & \\
& 0 & & & \ddots & a_{n} & \\
& & & & & & 1
\end{array}\right)
$$

for some $0<a_{1} \leqslant \cdots \leqslant a_{n}$. In particular every $\mathscr{T}$-metric is an $\mathscr{S}$-metric.

(2.8) Lemma. (i) Two left-invariant metrics $g$ and $g^{\prime}$ on the simply-connected Heisenberg group $H_{n}$ are isometric if and only if $g^{\prime}=\Phi^{*} g$ for some $\Phi \in \operatorname{Aut}\left(H_{n}\right)$.

(ii) Each left-invariant metric on $H_{n}$ is isometric to a unique $\mathscr{T}$-metric.

Proof. (i) is a special case of a result of [6].

(ii) Let $g$ be a left-invariant metric. By Lemma 3.5 of [5], there exists a $g$-orthonormal basis $\left\{X_{1}^{\prime}, \cdots, X_{n}^{\prime}, Y_{1}^{\prime}, \cdots, Y_{n}^{\prime}, Z^{\prime}\right\}$ of $\mathfrak{h}_{n}$ and $d_{1} \geqslant \cdots \geqslant d_{n}$ $\in \mathbf{R}^{+}$such that

$$
\left[X_{i}^{\prime}, Y_{i}^{\prime}\right]=d_{i}^{2} Z^{\prime}
$$

Thus the linear map $\varphi$ on $\mathfrak{h}_{n}$ which sends $X_{i}^{\prime}$ to $d_{i} X_{i}, Y_{i}^{\prime}$ to $d_{i} Y_{i}$, and $Z^{\prime}$ to $Z$ is the differential of an automorphism $\Phi$ of $H_{n}$. The metric $\Phi^{*} g$ is a $\mathscr{T}$-metric isometric to $g$ with $a_{i}=d_{i}^{-2}, 1 \leqslant i \leqslant n$. The uniqueness of the $\mathscr{T}$-metric is an easy consequence of (i) and the fact that an orthonormal basis for a $\mathscr{T}$-metric satisfies the bracket relations (2.9) with $d_{i}=a_{i}^{-2}$. 
(2.10) Uniform discrete subgroups. For $\mathscr{L}$ a lattice of maximal rank in $\mathbf{R}^{2 n}$ and for $c \in \mathbf{Z}^{+}$, let

$$
\Gamma(\mathscr{L}, c)=\left\{h(x, y, t) \in H_{n}:(x, y) \in \mathscr{L}, t \in c \mathbf{Z}\right\} .
$$

By (2.2), $\Gamma(\mathscr{L}, c)$ is a subgroup of $H_{n}$ if and only if

$$
x \cdot y^{\prime} \in c \mathbf{Z}, \quad \text { whenever }(x, y),\left(x^{\prime}, y^{\prime}\right) \in \mathscr{L} \text {. }
$$

Since $H_{n}$ is diffeomorphic to $\mathbf{R}^{2 n} \times \mathbf{R}$, we have that $\Gamma(\mathscr{L}, c)$ is a uniform discrete subgroup of $H_{n}$ whenever (2.11) holds. A uniform discrete subgroup $\Gamma$ of $H_{n}$ will be called a $\mathscr{T}$-group if $\Gamma$ is of the form $\Gamma=\Gamma(\mathscr{L}, c)$. We distinguish a subcollection of $\mathscr{T}$-groups, called $\mathscr{S}$-groups, as follows: For $r=\left(r_{1}, \cdots, r_{n}\right)$ $\in\left(\mathbf{Z}^{+}\right)^{n}$ such that $r_{i}$ divides $r_{i+1}, 1 \leqslant i \leqslant n-1$, let $\mathscr{L}_{r}=r_{1} \mathbf{Z} \times \cdots \times r_{n} \mathbf{Z} \times$ $\mathbf{Z}^{n}$ and let $\Gamma_{r}=\Gamma\left(\mathscr{L}_{r}, 1\right)$. Then a group $\Gamma$ is called an $\mathscr{S}_{\text {-group if }} \Gamma=\Gamma_{r}$ for some such $r$.

(2.12) Example. In $\S 6$ we will consider $\mathscr{T}$-groups for which

$$
\mathscr{L}=r_{1} \mathbf{Z} \times \cdots \times r_{n} \mathbf{Z} \times s_{1} \mathbf{Z} \times \cdots \times s_{n} \mathbf{Z}
$$

for some $r_{i}, s_{i} \in \mathbf{R}^{+}$. For such $\mathscr{L}$, condition (2.11) states that $\Gamma(\mathscr{L}, c)$ is a $\mathscr{T}$-group if and only if $r_{i} s_{i} \in d \mathbf{Z}, 1 \leqslant i \leqslant n$. We will call $\Gamma(\mathscr{L}, c)$ a rectilinear $\mathscr{T}$-group. One can check that $\Gamma(\mathscr{L}, c)$ is conjugate under $\operatorname{Aut}\left(H_{n}\right)$ to the $\mathscr{S}$-group $\Gamma_{\tilde{r}}$, where

$$
\tilde{r}=\left(r_{(1)} s_{(1)} / c, \cdots, r_{(n)} s_{(n)} / c\right)
$$

(the parentheses around the subscripts indicate that the $r_{i} s_{i}$ have been reordered so that $\left.r_{(i)} s_{(i)} \leqslant r_{(i+1)} s_{(i+1)}, 1 \leqslant i \leqslant n-1\right)$. By Lemma 2.13 below, it follows that two rectilinear $\mathscr{T}$-groups $\Gamma(\mathscr{L}, c)$ and $\Gamma\left(\mathscr{L}^{\prime}, c^{\prime}\right)$ are isomorphic as abstract groups if and only if

$$
r_{i}^{\prime} s_{i}^{\prime} / c^{\prime}=r_{\sigma(i)} s_{\sigma(i)} / c, \quad 1 \leqslant i \leqslant n,
$$

for some permutation $\sigma$ on $\{1, \cdots, \mathrm{n}\}$.

(2.13) Lemma [5, Theorem 2.4]. Every uniform discrete subgroup of $H_{n}$ is

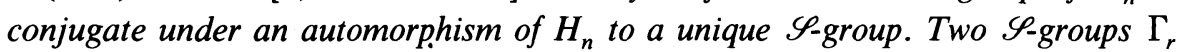
and $\Gamma_{r^{\prime}}$ are isomorphic as abstract groups if and only if $r=r^{\prime}$.

Definition. We let $\mathscr{S}$ (respectively $\mathscr{T}$ ) be the set of all Riemannian Heisenberg manifolds $\left(\Gamma \backslash H_{n}, g\right)$ such that $g$ is an $\mathscr{S}_{\text {-metric (respectively, a }}$ $\mathscr{T}$-metric) and $\Gamma$ is an $\mathscr{S}$-group (respectively, a $\mathscr{T}$-group).

(2.14) Proposition. Let $M=\left(\Gamma_{r} \backslash H_{n}, g\right) \in \mathscr{S}$ with $g$ given by (2.6). Then there exists $\Phi \in \operatorname{Aut}\left(H_{n}\right)$ of the form

$$
\Phi=\left(\begin{array}{cc} 
& 0 \\
d^{1 / 2} \alpha & \vdots \\
& 0 \\
0 \cdots 0 & \varepsilon(\alpha) d
\end{array}\right)
$$


for some $\alpha \in \widetilde{\operatorname{Sp}}(n, \mathbf{R})$ (see (2.4)) such that $\left(\Phi\left(\Gamma_{r}\right) \backslash H_{n},\left(\Phi^{-1}\right)^{*} g\right)$ is an element of $\mathscr{T}$ isometric to $M$. (Here $\Phi\left(\Gamma_{r}\right)=\Gamma(\mathscr{L}, d)$ for some $\mathscr{L}$.) Conversely, if $\left(\Gamma \backslash H_{n}, g\right) \in \mathscr{T}$ with $\Gamma=\Gamma(\mathscr{L}, c)$, then $\left(\Phi^{-1}(\Gamma) \backslash H_{n}, \Phi^{*} g\right)$ is an isometric element of $\mathscr{S}$ for some $\Phi$ of the form (2.15) with $d=c$.

Proof. For the first statement, Lemma 2.8 guarantees the existence of a $\mathscr{T}$-metric $g^{\prime}$ and an automorphism $\Phi$ such that $g=\Phi^{*} g^{\prime}$. In view of (2.4) and the expressions (2.6) and (2.7) for $g$ and $g^{\prime}$, we see that $\Phi$ is necessarily of the form (2.15). Trivially $\Phi\left(\Gamma_{r}\right)$ is a $\mathscr{T}$-group and the first statement follows. The second statement is proved similarly using Lemma 2.13 .

(2.16) Proposition. Every Riemannian Heisenberg manifold is isometric to an element of $\mathscr{T}$. Two elements $\left(\Gamma \backslash H_{n}, g\right)$ and $\left(\Gamma^{\prime} \backslash H_{n}, g^{\prime}\right)$ of $\mathscr{T}$ are isometric if and only if $g=g^{\prime}$ and there exists $\Phi \in \operatorname{Aut}\left(H_{n}\right)$ such that $g=\Phi^{*} g$ and $\Gamma^{\prime}=\Phi(\Gamma)$. Necessarily $\Phi$ is of the form (2.15) with $d=1$.

Proof. Proposition 2.16 with $\mathscr{T}$ replaced by $\mathscr{S}$ is proved in [5, Theorem 2.7]. Thus the first statement of Proposition 2.16 follows from Proposition 2.14. The proof of the second statement is the same as the proof of the analogous statement for $\mathscr{S}$ in [5] and is based on Lemma 2.8 .

(2.17) Notation. Given an element $\left(\Gamma \backslash H_{n}, g\right)$ of either $\mathscr{S}$ or $\mathscr{T}$ (note that in either case $\Gamma$ is of the form $\Gamma(\mathscr{L}, c)$ and $g$ of the form (2.6)), we associate to $\left(\Gamma \backslash H_{n}, g\right)$ the torus

$$
T\left(\mathscr{L}, g_{0}\right)=\left(\mathscr{L} \backslash \mathbf{R}^{2 n}, g_{0}\right),
$$

where the positive-definite matrix $g_{0}$ is viewed as a flat metric on $\mathbf{R}^{2 n}$.

(2.18) Proposition. If the element $\left(\Gamma \backslash H_{n}, g\right)$ of $\mathscr{T}$ is isometric to $\left(\Gamma_{r} \backslash H_{n}, g^{\prime}\right) \in \mathscr{S}$, then the associated tori are isometric.

Proof. By Propositions 2.14 and 2.16, we have $g^{\prime}=\Phi * g$ and $\Gamma=\Phi\left(\Gamma_{r}\right)$ for some $\Phi$ of the form (2.15). Denoting the submatrix $d^{1 / 2} \alpha$ of $\Phi$ by $\beta$, we see that $\mathscr{L}=\beta\left(\mathscr{L}_{r}\right)$ and $g_{0}^{\prime}={ }^{t} \beta g_{0} \beta$. Hence $T\left(\mathscr{L}, g_{0}\right)$ is isometric to $T\left(\mathscr{L}_{r}, g_{0}^{\prime}\right)$.

We conclude this section with a description of the Ricci tensors of $\mathscr{T}$-metrics.

(2.19) Notation. (i) Given an $\mathscr{S}_{-}$(or, in particular a $\mathscr{T}$ ) metric $g$, we denote by $\langle$,$\rangle both the inner product on \mathfrak{h}_{n}$ defined by $g$ and the restriction of this inner product to the subspace $\mathbf{R}^{2 n}$ of $\mathfrak{h}_{n}$. (The latter is just the inner product defined by $g_{0}$.) The associated norms will be denoted by $\|\cdot\|$. Define a linear transformation $\eta$ of $\mathbf{R}^{2 n}$ by

$$
[X, Y]=\langle X, \eta Y\rangle Z
$$

( $Z$ is given by (2.3).) As shown in [5], $\eta$ is skew-symmetric relative to $\langle$, with matrix $g_{0}^{-1} J$ relative to the standard basis $\left\{X_{1}, \cdots, X_{n}, Y_{1}, \cdots, Y_{n}\right\}$ of $\mathbf{R}^{2 n}$. 
In particular if $g$ is a $\mathscr{T}$-metric, then $\eta$ has matrix

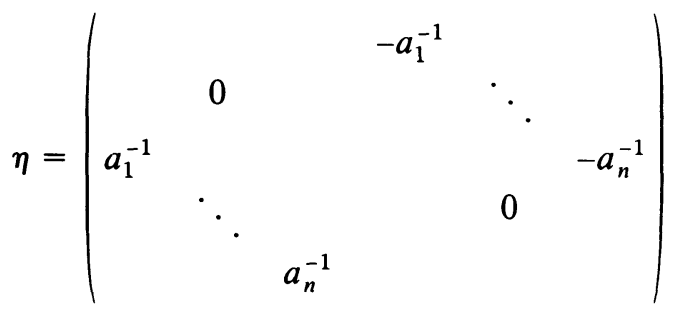

and $\eta^{2}=-g_{0}^{-2}$.

(ii) We denote the Ricci tensor of $g$ by $S_{g}$ and write $\operatorname{Ric}_{g}(X)=S_{g}(X, X)$. We will frequently delete the subscript $g$.

(2.22) Proposition. Let $g$ be a $\mathscr{T}$-metric as in (2.7). Note that the basis $\left\{a_{1}^{-1 / 2} X_{1}, \cdots, a_{n}^{-1 / 2} X_{n}, a_{1}^{-1 / 2} Y_{1}, \cdots, a_{n}^{-1 / 2} Y_{n}, Z\right\}$ of $\mathfrak{h}_{n}$ is g-orthonormal. Relative to this basis, the Ricci tensor $S_{g}$ is diagonal with matrix

where $\eta$ is given by (2.21).

$$
S_{g}=\left(\begin{array}{cc} 
& 0 \\
\frac{1}{2} \eta^{2} & \vdots \\
& 0 \\
0 \cdots 0 & \frac{1}{4} \operatorname{tr}\left(-\eta^{2}\right)
\end{array}\right),
$$

Proof. Let $A_{i}=a_{i}^{-1 / 2} X_{i}$ and $B_{i}=a_{i}^{-1 / 2} Y_{i}$. Using the facts that $\left[A_{i}, B_{i}\right]=$ $a_{i}^{-1} Z$ and that the covariant derivative for a left-invariant metric is given by $2\left\langle\nabla_{U} V, W\right\rangle=\langle[U, V], W\rangle+\langle[W, U], V\rangle+\langle[W, V], U\rangle$ when $U, V, W \in \mathfrak{h}_{n}$ (see [7, p. 48]), we find that

$$
\begin{gathered}
\nabla_{A_{i}}=\left(2 a_{i}\right)^{-1} B_{i} \wedge Z, \quad \nabla_{B_{i}}=\left(2 a_{i}\right)^{-1} A_{i} \wedge Z \\
\nabla_{Z}=-\frac{1}{2} \sum_{j=1}^{n} a_{j}^{-1} A_{j} \wedge B_{j} .
\end{gathered}
$$

Using $R_{g}(X, Y)=\left[\nabla_{x}, \nabla_{Y}\right]-\nabla_{[X, Y]}$, where $R_{g}$ is the curvature, we can then compute $R_{g}$ and $S_{g}$ to obtain the proposition.

\section{Comparison of the 0-spectra}

Throughout $\S \S 3-5, g$ will denote an arbitrary, but fixed, $\mathscr{T}$-metric, with diagonal entries $a_{1}, \cdots, a_{n}$ (see $\left.(2.7)\right)$ and $\Gamma=\Gamma(\mathscr{L}, c)$ and $\Gamma^{\prime}=\Gamma\left(\mathscr{L}^{\prime}, c^{\prime}\right)$ will denote $\mathscr{T}$-groups (see (2.10)). We will say $\Gamma$ and $\Gamma^{\prime}$ are p-isospectral relative to $g$ if the manifolds $\left(\Gamma \backslash H_{n}, g\right)$ and $\left(\Gamma^{\prime} \backslash H_{n}, g\right)$ are $p$-isospectral.

(3.1) Notation. (i) Recall that the dual lattice of the lattice $\mathscr{L}$ in $\mathbf{R}^{2 n}$ is the set of linear functionals $\tau$ on $\mathbf{R}^{2 n}$ such that $\tau(\mathscr{L}) \subset \mathbf{Z}$. We will view $\mathbf{R}^{2 n}$ as a subspace of $\mathfrak{h}_{n}$ and thus view elements of the dual lattice as linear functionals 
on $\mathfrak{h}_{n}$ which vanish on the center of $\mathfrak{h}_{n}$. We denote the dual lattices of $\mathscr{L}$ and $\mathscr{L}^{\prime}$ by $\mathscr{A}$ and $\mathscr{A}^{\prime}$, respectively.

(ii) Denote by $|\mathscr{L}|$ the volume of a fundamental domain of $\mathscr{L}$ relative to the standard metric on $\mathbf{R}^{2 n}$.

(iii) Given the metric $g$, we define as usual a map $\sharp$ : $\mathfrak{h}_{n}^{*} \rightarrow \mathfrak{h}_{n}$ by $\tau(X)=$ $\langle\sharp \tau, X\rangle$ and we let $b=\sharp^{-1}$. We again denote by $\langle$,$\rangle the inner product on \mathfrak{h}_{n}^{*}$ defined by $\langle\sigma, \tau\rangle=\langle \# \sigma, \sharp \tau\rangle$ and let $\|\cdot\|$ denote the associated norm.

In [5], sufficient conditions were given for two elements of $\mathscr{S}$ to be 0 isospectral. The "if" statement of the following theorem is the analogue for $\mathscr{T}$.

(3.2) Theorem. The groups $\Gamma(\mathscr{L}, c)$ and $\Gamma\left(\mathscr{L}^{\prime}, c\right)$ are 0 -isospectral relative to $g$ if and only if the following condition holds:

There exists a bijection $\theta: \mathscr{A} \rightarrow \mathscr{A}^{\prime}$ such that

$$
\|\theta \tau\|=\|\tau\| \text { for all } \tau \in \mathscr{A}
$$

(equivalently, the associated tori are 0 -isospectral).

(3.3) Remarks. (i) The spectrum of the torus $\left(\mathscr{L} \backslash \mathbf{R}^{2 n}, g_{0}\right)$ is given by $\left\{4 \pi^{2}\|\tau\|^{2}: \tau \in \mathscr{A}\right\}$ with the obvious multiplicities. Thus the parenthetical statement in the theorem is immediate.

(ii) If $\theta$ is $\mathbf{Z}$-linear, then the associated tori are not only 0 -isospectral but also isometric. I do not know whether any examples exist, of 0 -isospectral groups $\Gamma(\mathscr{L}, c)$ and $\Gamma\left(\mathscr{L}^{\prime}, c\right)$ for which no choice of $\theta$ in (P0) is Z-linear. One cannot automatically construct such examples using the known examples of isospectral, nonisometric tori (e.g. Milnor's example), since the lattices $\mathscr{L}$ and $\mathscr{L}^{\prime}$ are required to satisfy $(2.11)$.

(iii) Theorem 3.2 does not give necessary and sufficient conditions for any two elements $\left(\Gamma \backslash H_{n}, g\right)$ and $\left(\Gamma^{\prime} \backslash H_{n}, g^{\prime}\right)$ of $\mathscr{T}$ to be 0 -isospectral since the theorem assumes that $c=c^{\prime}$ and $g=g^{\prime}$. However [5] gives evidence suggesting, but not proving, that these conditions (translated into the $\mathscr{S}$ setting) may be necessary for 0 -isospectrality.

Proof of Theorem 3.2. We first show that the "if" statement follows from Theorem 4.1 of [5]. Write $\Gamma=\Gamma(\mathscr{L}, c)$ and $\Gamma^{\prime}=\Gamma\left(\mathscr{L}^{\prime}, c\right)$. Let $\tilde{M} \equiv$ $\left(\Gamma_{r} \backslash H_{n}, \tilde{g}\right)$ and $\tilde{M}^{\prime} \equiv\left(\Gamma_{r} \backslash H_{n}, \tilde{g}^{\prime}\right)$ be elements of $\mathscr{S}$ isometric, respectively, to $M \equiv\left(\left(\Gamma \backslash H_{n}, g\right)\right.$ and $M^{\prime} \equiv\left(\Gamma^{\prime} \backslash H_{n}, g\right)$ (see Proposition 2.14). We caution that $\tilde{g}$ need not equal $\tilde{g}^{\prime}$ although, viewed as metrics on the simplyconnected manifold $H_{n}, \tilde{g}$ and $\tilde{g}^{\prime}$ are isometric. Theorem 4.1 of [5] states that the following four conditions are sufficient for $\tilde{M}$ and $\tilde{M}^{\prime}$ to be 0 -isospectral.

(a) the $(2 n+1,2 n+1)$ entries of the matrices $\tilde{g}$ and $\tilde{g}^{\prime}$ coincide;

(b) $r_{1} \cdots r_{n}=r_{1}^{\prime} \cdots r_{n}^{\prime}$;

(c) $\tilde{g}$ and $\tilde{g}^{\prime}$ are isometric metrics on $H_{n}$;

(d) the associated tori are isometric. 
(We have rephrased (c) and (d).) From Proposition 2.14, we see that the $(2 n+1,2 n+1)$ entry of $\tilde{g}$ is $c^{2}$. Thus (a) is equivalent to our condition $c=c^{\prime}$. By Lemma 2.8, (c) is equivalent to our condition that the $\mathscr{T}$-metrics be identical, i.e. $g=g^{\prime}$. Condition (d) is stronger than (P0), but only the weaker condition that the associated tori be 0 -isospectral was used in the proof given in [5]. This weaker condition is equivalent, by Proposition 2.18, to (P0). Finally, (b) states that $\left|\mathscr{L}_{r}\right|=\left|\mathscr{L}_{r}^{\prime}\right|$. This condition is actually a consequence of (a), (b), and (d), since the volume of the torus associated to $\tilde{M}$ is $\left|\mathscr{L}_{r}\right|\left|\operatorname{det}\left(g_{0}\right)\right|^{1 / 2}$. (Recall that 0 -isospectral manifolds have the same volume, so weakening (d) does not affect (b).) This completes the proof of sufficiency of (P0).

In order to prove the necessity of (P0), we need to look at the right action of $H_{n}$ on $\Gamma \backslash H_{n}$.

(3.4) Notation. Let $\rho_{\Gamma}$ (or just $\rho$ ) denote the right action of $H_{n}$ on $L^{2}\left(\Gamma \backslash H_{n}\right)$. For $\tau$ in the dual lattice $\mathscr{A}$ of $\mathscr{L}$ define the character $f_{\tau}: H_{n} \rightarrow H_{n}$ by

$$
f_{\tau}(h)=\exp (2 \pi \sqrt{-1}(\log h)) .
$$

As discussed in [5], $f_{\tau} \in L^{2}\left(\Gamma \backslash H_{n}\right)$ and

$$
\rho(h) f_{\tau}=f_{\tau}(h) f_{\tau}
$$

for all $h \in H_{n}$. Thus $\mathbf{R} f_{\tau}$ is $\rho$-invariant.

Let

Then

$$
\mathscr{H}_{\Gamma, 1}=\bigoplus_{\tau \in \mathscr{A}} \mathbf{R} f_{\tau}
$$

$$
L^{2}\left(\Gamma \backslash H_{n}\right)=\mathscr{H}_{\Gamma, 1} \oplus \mathscr{H}_{\Gamma, 2},
$$

where $\mathscr{H}_{\Gamma, 2}$ consists of all irreducible invariant subspaces of infinite dimension.

(3.8) Lemma. Let $\Gamma=\Gamma(\mathscr{L}, c)$ and $\Gamma^{\prime}=\Gamma\left(\mathscr{L}^{\prime}, c\right)$.

(i) If $|\mathscr{L}|=\left|\mathscr{L}^{\prime}\right|$, then $\mathscr{H}_{\Gamma, 2}$ is g-equivalent to $\mathscr{H}_{\Gamma^{\prime}, 2}$.

(ii) The spectrum of the Laplacian of $\left(\Gamma \backslash H_{n}, g\right)$ acting on $\mathscr{H}_{\Gamma, 1}$ is the 0 -spectrum of the associated torus $\left(\mathscr{L} \backslash \mathbf{R}^{2 n}, g_{0}\right)$.

Proof. Part (i) of the lemma follows from [5, Lemma 3.7(b)] and the proof of Theorem 3.3 in [5]. (The proofs there use conditions (a), (b), and (c) discussed above.) The elementary result (ii) is also contained in the proof of Theorem 3.3 of [5].

We can now prove the necessity of (P0). Assume $\left(\Gamma \backslash H_{n}, g\right)$ is 0 -isospectral to $\left(\Gamma^{\prime} \backslash H_{n}, g\right)$. Then the two manifolds have the same volume. But one easily computes the volume of $\left(\Gamma \backslash H_{n}, g\right)$ to be $|\mathscr{L}| c \mid$ det $\left.g\right|^{1 / 2}$. Consequently $|\mathscr{L}|=$ $\left|\mathscr{L}^{\prime}\right|$ and by Lemma 3.8(i), we have that $\mathscr{H}_{\Gamma, 2}$ is $g$-equivalent to $\mathscr{H}_{\Gamma^{\prime}, 2}$. Hence, 
the Laplacians restricted to these subspaces are isospectral. By (3.7), the Laplacians acting on $\mathscr{H}_{\Gamma, 1}$ and $\mathscr{H}_{\Gamma^{\prime}, 1}$ must also be isospectral, so Lemma 3.8(ii) shows that (P0) holds.

We remark that Lemma 3.8 and Theorem 1.2 give another proof of the sufficiency of (P0).

\section{Comparison of the 1-spectra}

Let $\Gamma$ be a $\mathscr{T}$-group. By (3.7) and the discussion in $\S 1$, the space of square-integrable 1 -forms is given by

$$
\left(\mathscr{H}_{\Gamma, 1} \otimes \mathfrak{h}_{n}^{*}\right) \oplus\left(\mathscr{H}_{\Gamma, 2} \otimes \mathfrak{h}_{n}^{*}\right)
$$

We denote by $\sum_{1}^{i}(\Gamma, g)$ the spectrum of the Laplacian of $\left(\Gamma \backslash H_{n}, g\right)$ acting on $\mathscr{H}_{\Gamma, i} \otimes \mathfrak{h}_{n}^{*}, i=1,2$.

(4.1) Lemma. Suppose $\Gamma \equiv \Gamma(\mathscr{L}, c)$ and $\Gamma^{\prime} \equiv \Gamma\left(\mathscr{L}^{\prime}, c\right)$ satisfy (P0). Then $\Gamma$ is 1-isospectral to $\Gamma^{\prime}$ relative to $g$ if and only if $\Sigma_{1}^{1}(\Gamma, g)=\Sigma_{1}^{1}\left(\Gamma^{\prime}, g\right)$.

Proof. Condition (P0) implies that $|\mathscr{L}|=\left|\mathscr{L}^{\prime}\right|$ since isospectral tori have the same volume. Hence the lemma follows from Lemma 3.8(i) and Theorem 1.2 .

(4.2) Lemma [5]. Let $\Gamma=\Gamma(\mathscr{L}, c)$. For $\tau$ in the dual lattice $\mathscr{A}$ of $\mathscr{L}$, let

$$
\begin{gathered}
\alpha(\tau)=4 \pi^{2}\|\tau\|^{2}, \\
\beta_{ \pm}(\tau)=\alpha(\tau)+A+\left[A^{2}-8 \pi^{2} \operatorname{Ric}(\sharp \tau)\right]^{1 / 2},
\end{gathered}
$$

where $A=2 \operatorname{Ric}(Z)$. Then $\Sigma_{1}^{1}(\Gamma, g)$ is the collection of numbers $\lambda$ of the form $\lambda=\alpha(\tau)$ or $\lambda=\beta_{ \pm}(\tau)$ for some $\tau \in \mathscr{A}_{r}$. $\lambda$ occurs in $\Sigma_{1}^{1}(r, g) 2 n-1$ times for each $\tau \in \mathscr{A}$ such that $\lambda=\alpha(\tau)$ and once for each $\tau \in \mathscr{A}$ such that $\lambda=\beta_{+}(\tau)$ or $\lambda=\beta_{-}(\tau)$.

This lemma is the translation of Proposition A.4 of [5] to the $\mathscr{T}$-setting. We have used Proposition A.4 of [5] to the $\mathscr{T}$-setting. We have used Proposition 2.22 to convert expressions involving $\eta$ to expressions involving the Ricci curvature.

Lemmas 4.1 and 4.2 yield:

(4.3) Theorem. Suppose $\Gamma(\mathscr{L}, c)$ and $\Gamma\left(\mathscr{L}^{\prime}, c\right)$ are 0 -isospectral relative to g. If the bijection $\theta$ in (P0) can be chosen so that

$$
\operatorname{Ric}(\sharp \theta \tau)=\operatorname{Ric}(\sharp \tau) \text { for all } \tau \in \mathscr{A},
$$

then $\Gamma(\mathscr{L}, c)$ and $\Gamma\left(\mathscr{L}^{\prime}, c\right)$ are 1-isospectral relative to $g$.

It seems likely that (P1) is a necessary as well as sufficient condition for the manifolds to be 1-isospectral. We at least have: 
(4.4) Theorem. In addition to the hypotheses of Theorem 4.3, assume that the entries $a_{1}, \cdots, a_{n}$ of $g$ are algebraic and that all elements of $\mathscr{L}$ and $\mathscr{L}^{\prime}$ are linear combinations with algebraic coefficients of the standard basis vectors of $\mathbf{R}^{2 n}$. Then $\Gamma(\mathscr{L}, c)$ is 1-isospectral to $\Gamma\left(\mathscr{L}^{\prime}, c\right)$ if and only if some choice of $\theta$ satisfying (P0) also satisfies (P1).

Proof. We use the notation of Lemma 4.2. For any choice of $\theta$ in (P0), we have $\alpha(\tau)=\alpha(\theta \tau)$. We will show that if $\beta_{\varepsilon}(\tau)=\beta_{\varepsilon^{\prime}}\left(\tau^{\prime}\right)$ for some $\tau \in \mathscr{A}$, $\tau^{\prime} \in \mathscr{A}^{\prime}$, and $\varepsilon, \varepsilon^{\prime} \in\{+,-\}$, then $\|\tau\|=\left\|\tau^{\prime}\right\|$ and $\operatorname{Ric}(\sharp \tau)=\operatorname{Ric}\left(\sharp \tau^{\prime}\right)$. The theorem will follow.

Assume $\beta_{\varepsilon}(\tau)=\beta_{\varepsilon^{\prime}}\left(\tau^{\prime}\right)$. Set $B=4\|\tau\|^{2}, B^{\prime}=4\left\|\tau^{\prime}\right\|^{2}, C=-8 \operatorname{Ric}(\sharp \tau)$, and $C^{\prime}=-8 \operatorname{Ric}\left(\# \tau^{\prime}\right)$. The hypotheses of the theorem together with Proposition 2.22 imply that $A, B, B^{\prime}, C$, and $C^{\prime}$ are algebraic. Eliminating radicals in the equation

$$
B \pi^{2}+\varepsilon\left[A^{2}+C \pi^{2}\right]^{1 / 2}=B^{\prime} \pi^{2}+\varepsilon^{\prime}\left[A^{2}+C^{\prime} \pi^{2}\right]^{1 / 2},
$$

we obtain $P(\pi)=0$, where $P$ is a nontrivial polynomial with algebraic coefficients and leading coefficient $\left(B-B^{\prime}\right)^{4}$. Consequently $B=B^{\prime}$ and hence $C=C^{\prime}$, proving the theorem.

\section{Comparison of the $p$-spectra}

(5.1) Notation. For $g$ a $\mathscr{T}$-metric as in (2.7), let $0<b_{1}<\cdots<b_{k}$ denote the distinct $a_{j}$ 's and let

$$
V_{i}=\operatorname{span}_{\mathbf{R}}\left\{X_{j}, Y_{j}: a_{j}=b_{i}\right\} .
$$

Thus $\mathbf{R}^{2 n} \cong V_{1} \oplus \cdots \oplus V_{k}$. For $\tau \in\left(\mathbf{R}^{2 n}\right)^{*}$, we write $\tau=\tau_{1}+\cdots+\tau_{k}$ with $\#\left(\tau_{i}\right) \in V_{i}$.

(5.2) Theorem. Suppose $\Gamma \equiv \Gamma(\mathscr{L}, c)$ and $\Gamma^{\prime} \equiv \Gamma\left(\mathscr{L}^{\prime}, c\right)$ are 0 -isospectral with respect to $\mathrm{g}$. If the bijection $\theta$ in (P0) can be chosen so that

$$
\left\|(\theta \tau)_{i}\right\|=\left\|\tau_{i}\right\| \quad \text { for all } \tau \in \mathscr{A} \text { and } i=1, \cdots, k,
$$

then $\Gamma$ is $\rho$-isospectral to $\Gamma^{\prime}$ relative to $g$ for $p=0,1,2, \cdots, 2 n+1$.

The proof is based on the following lemma. We use Notation 3.4.

(5.3) Lemma. Let $\tau$ and $\sigma$ be linear functionals on $\mathfrak{h}_{n}$ vanishing on $\mathbf{R Z}$. Then $\mathbf{R} f_{\tau}$ is g-equivalent to $\mathbf{R} f_{\sigma}$ if and only if $\left\|\tau_{i}\right\|=\left\|\boldsymbol{\sigma}_{i}\right\|, 1 \leqslant i \leqslant k$.

Proof. On $\mathbf{R} f_{\tau}$, the right action $\rho(h)$ of $h \in H_{n}$ is just multiplication by $\exp \left[2 \pi \sqrt{-1} \tau(\log (h))(\operatorname{see}(3.5))\right.$. For $\Phi \in \operatorname{Aut}\left(H_{n}\right)$, the action $\rho \circ \Phi(h)$ of $\mathbf{R} f_{\sigma}$ is multiplication by $\exp \left[2 \pi \sqrt{-1} \sigma \circ \Phi_{*}(\log (h))\right]$. Thus $\mathbf{R} f_{\tau}$ is g-equivalent to $\mathbf{R} f_{\sigma}$ if and only if $\tau=\Phi *_{\sigma}$ for some $\Phi \in \operatorname{Aut}\left(H_{n}\right)$ satisfying $\Phi^{*} g=g$. The condition $\Phi^{*} g=g$ implies $\Phi_{*} V_{i}=V_{i}, 1 \leqslant i \leqslant k$, and hence the "only if" 
statement of the lemma follows. Conversely, if $\left\|\tau_{i}\right\|=\left\|\sigma_{i}\right\|, 1 \leqslant i \leqslant k$, set $A_{i}=\sharp \tau_{i}$ and $A_{i}^{\prime}=\sharp \sigma_{i}$. Note that $\left\|A_{i}\right\|=\left\|A_{i}^{\prime}\right\|$. After multiplying by a scalar, we may assume $\left\|A_{i}\right\|=\left\|A_{i}^{\prime}\right\|=1$. Since $V_{i}$ is the $b_{i}^{-2}$ eigenspace of $\eta$ (see Notation 2.19), we may complete $A_{i}$ and $A_{i}^{\prime}$ to ordered orthonormal bases $\mathscr{B}_{i}$ and $\mathscr{B}_{i}^{\prime}$, respectively, of $V_{i}$ such that $\left.\eta\right|_{V_{i}}$ has matrix

$$
\left(\begin{array}{ccccc}
0 & -b_{i}^{-1} & & & \\
b_{i}^{-1} & 0 & & 0 & \\
& & \ddots & & \\
& 0 & & 0 & -b_{i}^{-1} \\
& & & b_{i}^{-1} & 0
\end{array}\right)
$$

relative to both $\mathscr{B}_{i}$ and $\mathscr{B}_{i}^{\prime}$. It follows from (2.20) that the linear map $\varphi$ : $\mathfrak{h}_{n} \rightarrow \mathfrak{h}_{n}$ which maps $Z$ to $Z$ and elements of $\mathscr{B}_{i}$ to the corresponding elements of $\mathscr{B}_{i}^{\prime}, 1 \leqslant i \leqslant k$, is the differential of an automorphism $\Phi$ satisfying $\Phi * g=g$ and $\tau=\Phi^{*} \sigma$. The lemma is proved.

Theorem 5.2 follows from Lemma 5.3, Lemma 3.8(i) (recall that (P0) implies $\left.|\mathscr{L}|=\left|\mathscr{L}^{\prime}\right|\right)$, and Theorem 1.2.

We conclude this section by comparing conditions (P1) and (P2).

(5.4) Proposition. Suppose $\theta$ satisfies (P0). Then

(i) (P2) implies (P1).

(ii) If $\boldsymbol{\theta}$ is $\mathbf{Z}$-linear, then (P2) is equivalent to (P1).

Proof. For $\tau \in\left(\mathbf{R}^{2 n}\right)^{*}$,

$$
\operatorname{Ric}(\# \tau)=-\frac{1}{2} \sum_{i=1}^{k} b_{i}^{-2}\left\|\tau_{i}\right\|^{2}
$$

by Proposition 2.22 and Notation 5.1. This proves (i), and (ii) is easily verified.

(5.5) Remark. It seems likely that if $\Gamma(\mathscr{L}, c)$ and $\Gamma\left(\mathscr{L}^{\prime}, c\right)$ are both 0 - and 1 -isospectral relative to $g$, then they are also $p$-isospectral for all $p$. To prove this conjecture, one would have to first prove that (P1) is a necessary condition for the groups to be 1-isospectral even without the additional hypotheses of Theorem 4.4, and that (P2) and (P1) are equivalent even when $\theta$ is not Z-linear.

\section{Examples}

Let $g$ be a fixed $\mathscr{T}$-metric with diagonal entries $a_{1}, \cdots, a_{n}$ and let $\Gamma=$ $\Gamma(\mathscr{L}, c)$ and $\Gamma^{\prime}=\Gamma\left(\mathscr{L}^{\prime}, c\right)$ be rectilinear $\mathscr{T}$-groups as in Example 2.12. Thus $\mathscr{L}=r \mathbf{Z}^{n} \times s \mathbf{Z}^{n}$ and $\mathscr{L}^{\prime}=r^{\prime} \mathbf{Z}^{n} \times s^{\prime} \mathbf{Z}^{n}$ for some $r, s, r^{\prime}, s^{\prime} \in\left(\mathbf{R}^{+}\right)^{n}$, where we write $r \mathbf{Z}^{n}$ for $r_{1} \mathbf{Z} \times \cdots \times r_{n} \mathbf{Z}$. 
(6.1) Theorem. Let $g, \Gamma$, and $\Gamma^{\prime}$ be as above. Then $\Gamma^{\prime}$ is 0 -isospectral to $\Gamma$ if and only if the 2 -tuple

$$
\left(a_{1}\left(r_{1}^{\prime}\right)^{2}, \cdots, a_{n}\left(r_{n}^{\prime}\right)^{2}, a_{1}\left(s_{1}^{\prime}\right)^{2}, \cdots, a_{n}\left(s_{n}^{\prime}\right)^{2}\right)
$$

is a permutation of $\left(a_{1} r^{2}, \cdots, a_{n} r_{n}^{2}, a_{1} s_{1}^{2}, \cdots, a_{n} s_{n}^{1}\right)$.

(6.2) Theorem. In the above notation, assume that $a_{i}, r_{i}, s_{i}, r_{i}^{\prime}$, and $s_{i}^{\prime}$ are algebraic, $1 \leqslant i \leqslant n$, and assume $\Gamma^{\prime}$ is 0 -isospectral to $\Gamma$. Let $b_{1} \leqslant \cdots \leqslant b_{k}$ be the distinct $a_{i}$ 's and set

$$
\mathscr{J}_{i}=\left\{j \in\{1, \cdots, n\}: a_{j}=b_{i}\right\} .
$$

Then the following are equivalent:

(a) $\Gamma^{\prime}$ is 1-isospectral to $\Gamma$.

(b) $\Gamma^{\prime}$ is p-isospectral to $\Gamma$ for all $p$.

(c) The permutation in Theorem 6.1 can be chosen so that entries $a_{j}\left(r_{j}^{\prime}\right)^{2}$ and $a_{j}\left(s_{j}^{\prime}\right)^{2}$ with $j \in \mathscr{J}_{i}$ are carried to entries with indices also in $\mathscr{J}_{i}, 1 \leqslant i \leqslant k$.

We will prove both theorems simultaneously. Let $\mathscr{B}_{0}^{*} \equiv\left\{\alpha_{1}, \cdots, \alpha_{n}\right.$, $\left.\beta_{1}, \cdots, \beta_{n}\right\}$ denote the basis of $\left(\mathbf{R}^{2 n}\right)^{*}$ dual to $\mathscr{B}_{0} \equiv\left\{X_{1}, \cdots, X_{n}, Y_{1}, \cdots, Y_{n}\right\}$. Then the dual lattice $\mathscr{A}$ of $\mathscr{L}$ has basis

$$
\mathscr{B}_{r, s}^{*}=\left\{\alpha_{i} / r_{i}, \beta_{i} / s_{i}: 1 \leqslant i \leqslant n\right\} .
$$

For $\tau \in\left(\mathbf{R}^{2 n}\right)^{*}$ expressed in terms of the basis $\mathscr{B}_{0}^{*}$, we have $\|\tau\|^{2}={ }^{t} \tau g_{0}^{-1} \tau$, where as usual $g_{0}$ is the upper left $2 n \times 2 n$ submatrix of $g$, so

$$
\left\|\alpha_{i} / r_{i}\right\|^{2}=\left(a_{i} r_{i}^{2}\right)^{-1},\left\|\beta_{i} / s_{i}\right\|^{2}=\left(a_{i} s_{i}^{2}\right)^{-1} \text {. }
$$

Hence, as in Notation 5.1,

$$
\mathscr{B}_{r, s}^{*} \cap V_{i}=\left\{\alpha_{j} / r_{j}, \beta_{j} / s_{j}: j \in \mathscr{J}_{i}\right\} .
$$

Note that the existence of a permutation as in Theorem 6.1 is equivalent to the existence of a Z-linear map $\theta: \mathscr{A}^{\prime} \rightarrow \mathscr{A}$ which satisfies (P0) and which maps $\mathscr{B}_{r, s}^{*}$ to $\mathscr{B}_{r^{\prime}, s^{\prime}}^{*}$. The map $\theta$ also satisfies condition (P2) if and only if the permutation satisfies the condition stated in Theorem 6.2(c). Thus in view of Theorems 3.2, 4.4, and 5.2, and Proposition 5.4, Theorems 6.1 and 6.2 will follow from the following:

Claim. If there exists a map $\tilde{\theta}$ satisfying (P0), then there exists a $\mathbf{Z}$-linear map $\theta$ which satisfies (P0) and which maps $\mathscr{B}_{r, s}^{*}$ to $\mathscr{B}_{r^{\prime}, s^{\prime}}^{*}$. Moreover if $\tilde{\theta}$ satisfies (P1), then $\theta$ can be chosen so that it also satisfies (P1).

To prove the claim, let

$$
d_{1}=\min \{\|\tau\|: \tau \in \mathscr{A}\}=\min \left\{\|\tau\|^{\prime}: \tau^{\prime} \in \mathscr{A}^{\prime}\right\} .
$$

(The second equality follows from the existence of $\tilde{\theta}$.) Since $\mathscr{B}_{r, s}^{*}$ and $\mathscr{B}_{r^{\prime}, s^{\prime}}^{*}$ are orthogonal lattice bases of $\mathscr{A}$ and $\mathscr{A}^{\prime}$, all the elements $\sigma_{1}, \cdots, \sigma_{l}$ of norm $d_{1}$ in $\mathscr{A}$ and $\boldsymbol{\sigma}_{1}^{\prime}, \cdots, \boldsymbol{\sigma}_{l}^{\prime}$ of norm $d_{1}$ in $\mathscr{A}^{\prime}$ belong to $\mathscr{B}_{r, s}^{*}$ and to $\mathscr{B}_{r^{\prime}, s^{\prime}}^{*}$, 
respectively. Let $A_{1}=\operatorname{span}_{\mathbf{z}}\left\{\sigma_{1}, \cdots, \sigma_{l}\right\}$. Define $\theta$ to be linear on $A_{1}$ and to agree with $\tilde{\theta}$ on $\sigma_{1}, \cdots, \sigma_{l}$. Since the standard basis $\mathscr{B}$ of $\mathfrak{h}_{n}$ diagonalizes the Ricci tensor and since the elements of $\mathscr{B}_{r, s}^{*}$ and $\mathscr{B}_{r^{\prime}, s^{\prime}}^{\prime}$ are multiplies of the elements of $\mathscr{B}_{0}^{*} \subset \mathscr{B}^{*}$, the map $\theta$ on $A_{1}$ satisfies condition (P1) if $\tilde{\theta}$ satisfies (P1).

Next let

$$
d_{2}=\min \left\{\|\tau\|: \tau \in \mathscr{A}-A_{1}\right\} .
$$

By making finitely many changes in $\tilde{\theta}$ (without affecting whether $\tilde{\theta}$ satisfies (P1)), we may assume that $\tilde{\theta}(\tau)=\theta(\tau)$ whenever $\tau \in A_{1}$ and $\|\tau\| \leqslant d_{2}$. Thus if $\tau \in \mathscr{A}-A_{1}$ and $\|\tau\|=d_{2}$, then $\tilde{\theta}(\tau) \in \mathscr{A}^{\prime}-\theta\left(A_{1}\right)$. Note moreover that if $\tau \in \mathscr{A}-A_{1}$ and $\|\tau\|=d_{2}$, then $\tau \in \mathscr{B}_{r, s}^{*}$ since $A_{1}$ is spanned by elements of the orthogonal basis $\mathscr{B}_{r, s^{*}}^{*}$. For the same reason, $\tilde{\theta}(\tau) \in \mathscr{B}_{r^{\prime}, s^{\prime}}^{*}$. Thus we may extend $\theta$ to a $\mathbf{Z}$-linear map on the sublattice $A_{2}$ of $\mathscr{A}$ spanned by $A_{1}$ and all elements of $\mathscr{B}_{r, s}^{*}$ of norm $d_{2}$. If $\tilde{\theta}$ satisfies (P1), then $\theta$ satisfies (P1) on $A_{2}$.

Continuing this construction for at most $2 n$ steps, we obtain the desired $\operatorname{map} \theta$.

This proves the claim and, as noted above, Theorems 6.1 and 6.2 follow.

We remark that Theorem 6.1 is just the statement that any two isospectral flat rectilinear tori are isometric.

(6.3) Examples. The following chart gives four pairs of 0 -isospectral Heisenberg manifolds. The manifolds $\left(\Gamma \backslash H_{n}, g\right)$ and $\left(\Gamma^{\prime} \backslash H_{n}, g\right)$ are specified as follows: In the column labeled $g$, we give the diagonal entries $a_{1}, \cdots, a_{n}$ of the $\mathscr{T}$-metric $g$. We have $\Gamma=\Gamma(\mathscr{L}, 1)$ and $\Gamma^{\prime}=\Gamma\left(\mathscr{L}^{\prime}, 1\right)$, where $\mathscr{L}=r \mathbf{Z}^{n}$ $\times s \mathbf{Z}^{n}$ and $\mathscr{L}^{\prime}=r^{\prime} \mathbf{Z}^{n} \times s^{\prime} \mathbf{Z}^{n}$. In the columns labeled $\mathscr{L}$ and $\mathscr{L}^{\prime}$, we specify the $2 n$-tuples $\left(r_{1}, \cdots, r_{n} ; s_{1}, \cdots, s_{n}\right)$ and $\left(r_{1}^{\prime}, \cdots, r_{n}^{\prime} ; s_{1}^{\prime}, \cdots, s_{n}^{\prime}\right)$, respectively. Thus $\left(\Gamma \backslash H_{n}, g\right)$ is completely specified by the entries in the columns $g$ and $\mathscr{L}$, and $\left(\Gamma^{\prime} \backslash H_{n}, g\right)$ is specified by the entries in columns $g$ and $\mathscr{L}^{\prime}$. In each case, Theorem 6.1 implies that the manifolds are 0-isospectral. We use Theorem 6.2 to test whether they are 1-isospectral (and consequently $p$-isospectral for all $p$ ). Finally the condition given at the end of Example 2.12 can be used to check whether the fundamental groups $\Gamma$ and $\Gamma^{\prime}$ are isomorphic.

\begin{tabular}{c|c|c|c|c|c}
$n$ & $g$ & $\mathscr{L}$ & $\mathscr{L}^{\prime}$ & 1 -isospectral & $\begin{array}{c}\text { fundamental } \\
\text { groups } \\
\text { isomorphic }\end{array}$ \\
\hline 2 & $(1,4)$ & $(4,2 ; 1,2)$ & $(4,1 / 2 ; 4,2)$ & no & no \\
2 & $(1,1)$ & $(2,1 ; 2,1)$ & $(2,2 ; 1,1)$ & yes & no \\
2 & $(1,4)$ & $(4,2 ; 1,1 / 2)$ & $(1,2 ; 1,2)$ & no & yes \\
3 & $(1,1,1)$ & $(2,4,3 ; 6,5,10)$ & $(3,2,5 ; 4,10,6)$ & yes & yes
\end{tabular}


In the first three examples, the information in the last two columns guarantees that $\left(\Gamma \backslash H_{n}, g\right)$ is not isometric to $\left(\Gamma^{\prime} \backslash H_{n}, g\right)$. In the fourth example, one can check directly that there is no automorphism $\Phi$ of $H_{n}$ such that $\Phi^{*} g=g$ and $\Phi(\Gamma)=\Gamma^{\prime}$ and hence that the manifolds are nonisometric (see Proposition 2.16).

Remarks. (i) In [3], the length spectra, i.e., the collection of lengths of closed geodesics, are compared for pairs of manifolds satisfying the condition (P0) of Theorem 3.2. If one ignores multiplicities, the pairs of manifolds are length-isospectral. The same conclusion holds if one defines the multiplicity of an element of the length spectrum to be the total number of closed geodesics of that length. (All multiplicities are then infinite.) However in many cases, including each of the pairs of manifolds in the chart above, the manifolds can be distinguished by the number of free homotopy classes of loops containing a geodesic of specified length. This gives an alternative proof that the manifolds in the fourth example above are not isometric.

(ii) Generalizing the notion of a rectilinear $\mathscr{T}$-group, one many study $\mathscr{T}$-groups for which $\mathscr{L}=\oplus_{i=1}^{k} \mathscr{L} \cap V_{i}$ (Notation 5.1). Set $\mathscr{L}_{i}=\mathscr{L} \cap V_{i}$. Note that $V_{i}+\mathbf{R} Z$ is an ideal in $\mathfrak{h}_{n}$ isomorphic to a Heisenberg algebra of lower dimension. Setting $M_{i}=\exp \left(V_{i}+\mathbf{R} Z\right)$, we have that $M_{i}$ is a simply-connected normal subgroup of $H_{n}$. Moreover, $M_{i} \cap \Gamma=\Gamma\left(\mathscr{L}_{i}, c\right)$, a $\mathscr{T}$-group in the Heisenberg group $M_{i}$. The $\mathscr{T}$-metric $g$ induces a $\mathscr{T}$-metric $g_{i}$ on $M_{i}$ given by

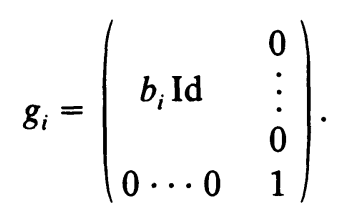

Thus the submanifold $\left(\left(M_{i} \cap \Gamma\right) \backslash M_{i}, g_{i}\right)$ is a Riemannian Heisenberg manifold of the type $\mathscr{T}$. We denote it by $M_{i}(\mathscr{L}, g)$. Moreover relative to the obvious orthonormal basis of $V_{i}+\mathbf{R} Z$, the Ricci tensor of $M_{i}(\mathscr{L}, g)$ is given by

$$
\left(\begin{array}{cc} 
& 0 \\
-\frac{1}{2} b_{i}^{-2} \mathrm{Id} & \vdots \\
0 \cdots 0 & \frac{1}{2} m_{i} b_{i}^{-2}
\end{array}\right),
$$

where $\operatorname{dim} V_{i}=2 m_{i}$. Thus we will call $M_{i}(\mathscr{L}, g)$ a submanifold of almost constant Ricci curvature.

Now suppose $\left(\Gamma \backslash H_{n}, g\right)$ and $\left(\Gamma^{\prime} \backslash H_{n}, g\right)$ are 0 -isospectral manifolds as in Theorem 3.2 and that $\mathscr{L}=\oplus_{i=1}^{k}: \mathscr{L} \cap V_{i}$ and $\mathscr{L}^{\prime}=\oplus_{i=1}^{k} \mathscr{L}^{\prime} \cap V_{i}$. In case 
$k=1$ (i.e. $a_{1}=\cdots=a_{n}$ ), the two manifolds themselves have almost constant Ricci curvature. Condition (P2) is trivially satisfied and hence they are $p$ isospectral for all $p$. If $k \geqslant 1$ and if $M_{i}(\mathscr{L}, g)$ is 0 -isospectral (and hence $p$-isospectral) to $M_{i}\left(\mathscr{L}^{\prime}, g\right)$ for $i=1, \cdots, k$, then it is an easy consequence of Theorems 3.2 and 5.2 that $\left(\Gamma \backslash H_{n}, g\right)$ and $\left(\Gamma^{\prime} \backslash H_{n}, g\right)$ are $p$-isospectral for all $p$. As a partial converse, if a $\mathbf{Z}$-linear choice of $\theta$ satisfies (P0) and (P1) (and so by Proposition 5.4, $\Gamma$ is $p$-isospectral to $\Gamma^{\prime}$ for all $\left.p\right)$, then $M_{i}(\mathscr{L}, g)$ is isospectral to $M_{i}\left(\mathscr{L}^{\prime}, g\right), 1 \leqslant i \leqslant k$.

(iii) Theorem 4.3 as well as (ii) above suggest ways of distinguishing geometrically the examples of Heisenberg manifolds which are $p$-isospectral for all $p$ from examples which are only 0 -isospectral. However we caution that for simply-connected Heisenberg manifolds $\left(H_{n}, g\right)$, the Ricci tensor is a complete isometry invariant. Hence our conditions involving only the Ricci tensor and the lattice vectors should not be expected to generalize immediately to other manifolds.

\section{References}

[1] D. M. Deturck \& C. S. Gordon, Isospectral deformations I: Riemannian structures on two-step nilspaces, preprint.

[2] P. B. Gilkey, On spherical space forms with meta-cyclic fundamental group which are isospectral but not equivariant cobordant, preprint.

[3] C. S. Gordon, The Laplace spectra versus the length spectra of Riemannian manifolds, Nonlinear Problems in Geometry (D. M. DeTurck, ed.), Contemporary Math., to appear.

[4] C. S. Gordon \& E. N. Wilson, Isospectral deformations of compact solvmanifolds, J. Differential Geometry 19 (1984) 241-256.

[5] The spectrum of the Laplacian on Riemannian Heisenberg manifolds, Michigan Math. J., to appear.

[6] _ Isometry groups of Riemannian solvmanifolds, preprint.

[7] S. Helgason, Differential geometry, Lie groups and symmetric spaces, Academic Press, New York, 1978.

[8] A. Ikeda, On spherical space forms which are isospectral but not isometric, J. Math. Soc. Japan 35 (1983) 437-444.

[9] V. K. Patodi, Curvature and the fundamental solution of the heat equation, J. Indian Math. Soc. 34 (1970) 269-285.

[10] T. Sunada, Riemannian coverings and isospectral manifolds, Ann. of Math. 121 (1985) $169-186$.

[11] M. F. Vignéras, Variétés Riemanniennes isospectrales et non isométriques, Ann. of Math. 112 (1980) 21-32.

WASHINGTON UNIVERSITY 